\title{
Some remarks on Birkhoff and Mather twist map theorems
}

\author{
A. KATOK \\ Department of Mathematics, University of Maryland, College Park, Maryland 20742, \\ USA
}

(Received 17 April 1982)

Abstract. A recent result of $\mathrm{J}$. Mather [1] about the existence of quasi-periodic orbits for twist maps is derived from an appropriately modified version of $G$. D. Birkhoff's classical theorem concerning periodic orbits. A proof of Birkhoff's theorem is given using a simplified geometric version of Mather's arguments. Additional properties of Mather's invariant sets are discussed.

\section{Notation}

Let

$$
A=S^{1} \times[0,1]=\{(\phi, r), \phi \in \mathbb{R} / \mathbb{Z}, 0 \leq r \leq 1\}
$$

be the standard annulus,

$$
S=\mathbb{R} \times[0,1]=\{(x, y), x \in \mathbb{R}, 0 \leq y \leq 1\}
$$

its universal covering, and $T: S \rightarrow S$ the unit translation

$$
T(x, y)=(x+1, y) \text {. }
$$

For any homeomorphism $f: A \rightarrow A$ its lift to $S$ is defined up to a power of $T$; conversely, if $F: S \rightarrow S$ and $F$ commutes with $T$ then $F$ is a lift of a homeomorphism of $A$. Let us write such an $F$ in the coordinate form:

$$
F(x, y)=\left(F_{1}(x, y), F_{2}(x, y)\right) \text {. }
$$

We shall call a homeomorphism $f: A \rightarrow A$ a twist homeomorphism (or a twist map) if it preserves orientation, preserves boundary components of $\boldsymbol{A}$ and if for a lift $F$ of $f$ and for any $x \in \mathbb{R}$ the function $F_{1}(x, y)$ is a strictly monotone function of $y$.

Obviously, all functions $F_{1}(x, y)$ for different $x$ must be either increasing or decreasing. According to that we may speak of right or left twist maps. If $f$ is a right twist map, then $f^{-1}$ is a left twist map and vice versa. For definiteness we shall always assume that $f$ is a right twist map.

We shall associate with a twist map $f$ the following objects.

Twist interval $\left[\alpha_{0}(f), \alpha_{1}(f)\right]$ where $\alpha_{0}(f)$ and $\alpha_{1}(f)$ are rotation numbers of $F$ restricted to $\mathbb{R} \times\{0\}$ and $\mathbb{R} \times\{1\}$ correspondingly. Twist interval is defined up to an integral translation. 
Twist modulus $\omega_{f}(r)$ which is defined for $0 \leq r \leq 1$ by

$$
\omega_{f}(r)=\min _{0 \leq x \leq 1} \min _{0 \leq y \leq 1-r} \min _{0}\left(F_{1}(x, y+r)-F_{1}(x, y), \hat{F}_{1}(x, y)-\hat{F}_{1}(x, y+r)\right)
$$

where $\hat{F}_{1}$ is the first coordinate for a lift of $f^{-1}$.

If $f$ is a twist map and in addition $f$ and $f^{-1}$ are Lipschitz maps and $\omega(r) \geq K r$ for some $K$ we shall call $f$ a Lipschitz twist map. For example, any $C^{1}$ diffeomorphism $f$ such that $\partial F_{1} / \partial y>0$ is a Lipschitz twist map.

\section{Birkhoff periodic orbits}

If $f^{q} z=z$ then $F^{q} w=T^{p} w$ for any lift $w$ of $z$ where the number $p$ is determined up to a multiple of $q$. Thus, the fraction $p / q$ is determined up to an integer. We shall call this fraction the rotation number of the periodic point $z$.

We shall call a point $z$ a Birkhoff point of type $(p, q)$ if for a lift $w$ of $z$ there exists a map

$$
\theta=(\psi, \rho): \mathbb{Z} \rightarrow S
$$

such that

$$
\theta(0)=w
$$

$\psi$ is a strictly monotone function,

$$
\begin{aligned}
& \theta(n+q)=T \theta(n), \\
& \theta(n+p)=F \theta(n) .
\end{aligned}
$$

We shall call the orbit of a Birkhoff point of type $(p, q)$ a Birkhoff periodic orbit of type $(p, q)$.

Obviously, any Birkhoff point of type $(p, q)$ is a periodic point with rotation number $p / q$. Moreover, if we denote for such a point $z$,

$$
f^{n} z=\left(\phi_{n}, r_{n}\right) \quad n=0, \ldots, q-1
$$

then all $\phi_{n}$ 's are different and lie on the circle in the same order as successive images of a point under rotations by $2 \pi p / q$. However, these two properties do not guarantee that $z$ is a Birkhoff point of type $(p, q)$. In particular, the assertion of proposition 1 below does not follow from those properties.

Let $\beta(r)$ be a common modulus of continuity for $f$ and $f^{-1}$. We can assume that $\beta$ is a concave function.

Proposition 1. Let $f$ be a twist map. There exists a concave monotone function $\gamma(r)$ positive for positive $r$ and depending only on $\omega_{f}$ and $\beta$ such that for every Birkhoff periodic orbit

$$
\left|\phi_{n}-\phi_{m}\right|<r
$$

implies that

$$
\left|r_{n}-r_{m}\right|<\gamma(r)
$$

If $f$ is a Lipschitz twist map then $\gamma(r)$ can be chosen as Lr for some constant $L$.

Proof. We shall work on the universal covering. Let us assume for definiteness that $\psi$ is an increasing function and let $m, n(m<n)$ be two integers, so that $\psi(m)<\psi(n)$. 
Let us assume that $\rho(n)<\rho(m)$ and denote

Since

$$
F(\psi(n), \rho(m))=(\tilde{\psi}, \tilde{\rho}) .
$$

$$
F(\psi(n), \rho(n))=(\psi(n+p), \rho(n+p))
$$

and $\rho(n)<\rho(m)$ we have by the twist condition

$$
\tilde{\psi}>\psi(n+p)+\omega_{f}(\rho(m)-\rho(n))
$$

On the other hand

$$
\psi(n+p)>\psi(m+p)>\tilde{\psi}-\beta(\psi(n)-\psi(m)) .
$$

From (1), (2) and (3) we have

$$
\omega_{f}(\rho(m)-\rho(n))<\beta(\psi(n)-\psi(m)) .
$$

Thus, we can put $\gamma=\tilde{\omega}^{-1} \circ \beta$ where $\tilde{\omega}$ is a strictly monotone convex function such that for $r>0$,

$$
0<\tilde{\omega}(r) \leq \omega_{f}(r) .
$$

If $\rho_{n}>\rho_{m}$ the argument goes the same way with $f^{-1}$ instead of $f$.

In the Lipschitz case we can take $\beta(r)=M r$ for some constant $M$ and $\tilde{\omega}(r)=K r$ so that $\gamma(r)=M K^{-1} r$.

\section{Mather sets}

We shall call a closed $f$-invariant set $E \subset A$ a Mather set if (i) $E$ intersects every interval $\{\phi\} \times[0,1]$ at most at one point, i.e., $E=$ graph $\Phi$ where $\Phi$ is a continuous function defined on a closed subset $K$ of $S^{1}$ with the values at [0,1]; (ii) $F$ preserves the order on the covering of $E$.

It is easy to see that $\left.f\right|_{E}$ preserves the natural cyclic order on $E$ and is topologically conjugate by an order-preserving homeomorphism to a restriction of a homeomorphism of the circle to the set $K$. In particular, the rotation number $\rho(E)$ is defined up to an integer.

Every closed subset of a Mather set is also a Mather set. In particular, every such set contains a minimal subset. It follows from standard Poincaré-Denjoy theory of circle homeomorphisms that there are exactly three sorts of minimal Mather sets. Namely

if $\rho(E)$ is rational and is equal to, say, $p / q$, then $E$ is a Birkhoff periodic orbit of type $(p, q)$;

if $\rho(E)$ is irrational, then $E$ is either a circle and $\left.f\right|_{E}$ is conjugate to the rotation by $\rho(E)$ or $E$ is a Cantor set and $\left.f\right|_{E}$ is conjugate to the minimal set for one of the so-called Denjoy counterexamples.

Proposition 2. For any Mather set $E$ the function $\Phi$ has modulus of continuity $\gamma$ described in proposition 1.

The proof of this proposition is a mere repetition of the proof of proposition 1 . For, in that proof the periodicity of the orbit is not important; only the preservation of order on the universal covering matters. 
Remark. We do not need proposition 2 in order to derive Mather's theorem from Birkhoff's theorem.

Let us recall the definition of Hausdorff topology on the space of all closed subsets of a compact metric space $X$. Let $U_{\varepsilon}(E)$ denote the open $\varepsilon$-neighbourhood of the set $E$ in $X$. The basis of neighbourhoods of $E$ in Hausdorff topology is formed by the sets

$$
V_{\varepsilon}(E)=\left\{F \subset X: F \subset U_{\varepsilon}(E), E \subset U_{\varepsilon}(F)\right\} .
$$

Proposition 3. (a) The set of all Mather sets for a twist homeomorphism is closed in Hausdorff topology.

(b) The rotation number $\rho(E)$ for a Mather set $E$ is continuous in Hausdorff topology.

Proof. Let $E_{n}=$ graph $\Phi_{n}$ where $\Phi_{n}: K_{n} \rightarrow[0,1]$ be a sequence of Mather sets for $f$ converging to a set $E$ in Hausdorff topology.

Let

$$
z=\left(\phi^{0}, r^{0}\right) \in E, z_{n}=\left(\phi^{n}, r^{n}\right) \in E_{n}, z_{n} \rightarrow z
$$

and let us fix a small $\varepsilon>0$. Since by proposition 2

$$
\begin{gathered}
E_{n} \cap\left[\phi^{n}-\varepsilon ; \phi^{n}+\varepsilon\right] \times S^{1} \subset\left\{(\phi, r): \phi^{n}-\varepsilon \leq \phi \leq \phi^{n}+\varepsilon,\right. \\
\left.r^{n}-\gamma\left(\left|r-r^{n}\right|\right) \leq r \leq r^{n}+\gamma\left(\left|r-r^{n}\right|\right)\right\},
\end{gathered}
$$

the convergence in Hausdorff topology guarantees that

$$
\begin{gathered}
E \cap\left[\phi^{0}-\varepsilon, \phi^{0}+\varepsilon\right] \times S^{1} \subset\left\{(\phi, r): \phi^{0}-\varepsilon \leq \phi \leq \phi^{0}+\varepsilon,\right. \\
\left.r^{0}-\gamma\left(\left|r-r^{0}\right|\right) \leq r \leq r^{0}+\gamma\left(\left|r-r^{0}\right|\right)\right\} .
\end{gathered}
$$

This implies condition (i) for the set $E$. Condition (ii) then follows immediately from this condition for $E_{n}$ and from the convergence. This proves $(a)$.

Let now $\rho$ be any limit point of the sequence $\rho\left(E_{n}\right)$; say $\rho\left(E_{n_{k}}\right) \rightarrow \rho$. Let us denote

$$
f^{m}\left(z_{n_{k}}\right)=\left(\phi_{m}^{k}, r_{m}^{k}\right), f^{m}(z)=\left(\phi_{m}, r_{m}\right) \text {. }
$$

In order to prove that $\rho(E)=\rho$ it is enough to show that the order of $\phi_{m}$ 's on the circle is the same as the order of successive rotations of a point by $2 \pi \rho$. For any fixed $N$ we can find $k(N)$ such that for $k \geq k(N)$ the order of the first $N$ successive rotations by $2 \pi \rho\left(E_{n_{k}}\right)$ is the same as for successive $2 \pi \rho$-rotations. By property (ii) for the sets $E_{n_{k}}$ the order of $\phi_{m}^{k}$ 's is the same as the order of successive $2 \pi \rho\left(E_{n_{k}}\right)$ rotations. But since all angles $\phi_{m}, m=0, \ldots, N-1$, are pairwise different, for sufficiently large $k$ the order of $\phi_{m}^{k}$ 's and $\phi_{m}$ 's for $m=0, \ldots, N-1$ coincide. Since $N$ can be chosen arbitrary large $\rho(E)=\rho$ and this proves $(b)$.

From this proposition and the compactness of the Hausdorff topology we obtain immediately

Corollary 1. The union of all Mather sets is a closed f-invariant set.

Let us denote this set $M(f)$. We shall discuss some of its properties later.

COROLLARY 2. If $f$ has a Birkhoff orbit of type $(p, q)$ for any rational number $p / q$ from the twist interval then $f$ also possesses a minimal Mather set with any irrational rotation number from the twist interval. 
The assertion of this corollary for area-preserving twist homeomorphism constitutes the statement of Mather's theorem [1]. Thus, we have reduced this theorem to the question about the existence of Birkhoff periodic orbits.

Problem. Are periodic orbits dense in $M(f)$ ?

\section{Birkhoff theorem}

THEOREM. Let $f$ be a twist homeomorphism preserving a measure positive on open sets and $p / q$ belong to the twist interval. Then $f$ has a Birkhoff periodic orbit of type $(p, q)$.

If in addition $f$ is a $C^{1}$ diffeomorphism and preserves a measure given by a positive smooth density then under the same assumptions f has two different Birkhoff periodic orbits of type $(p, q)$ which form together a Mather set.

Proof. For the notational convenience we shall work with the universal covering instead of the annulus. Let us denote $F_{1}(x, 0)=g_{0}(x)$ and $F_{1}(x, 1)=g_{1}(x)$. Let us consider the space of all non-decreasing maps $\phi: \mathbb{Z} \rightarrow \mathbb{R}$ such that

$$
\phi(n+q)=\phi(n)+1
$$

and

$$
\phi(n)+g_{0}(\phi(n)) \leq \phi(n+p) \leq \phi(n)+g_{1}(\phi(n))
$$

and identify every such map $\phi(n)$ with $\phi(n)+k$ for every integer $k$. We denote the factor space $\Phi_{p, q}$.

The natural compact topology on $\Phi_{p, q}$ comes from the embedding

$$
\Phi_{p, q} \rightarrow \mathbb{R}^{q} / \mathbb{Z} \simeq \mathbb{R}^{q-1} \times \mathbb{R} / \mathbb{Z},
$$

which assigns to a map $\phi$ the vector $(\phi(0), \ldots, \phi(q-1))$. The factorization in $\mathbb{P}^{q}$ is defined by the diagonal integer shifts

$$
\left(x_{1}, \ldots, x_{q}\right) \rightarrow\left(x_{1}+n, \ldots, x_{q}+n\right), n \in \mathbb{Z} .
$$

It is easy to see that the identification in $\Phi_{p, q}$ corresponds to this factorization. Compactness follows from (5).

It is easy to see that $\Phi_{p, q}$ is non-empty; moreover for every $x \in \mathbb{R}$ there is $\phi \in \Phi_{p, q}$ such that $\phi(0)=x$. For, let $g_{t}(x), 0 \leq t \leq 1$, be a monotone family of homeomorphisms of $\mathbb{R}$ connecting $g_{0}$ and $g_{1}$. Since $\alpha_{0}(f)<p / q$ and $\alpha_{1}(f)>p / q$ we have

$$
g_{0}^{q}(x)-x<p, g_{1}^{q}(x)-x>p
$$

so that for some $f \in[0,1], g_{t}^{q}(x)=x+p$. Then the map $\phi$ defined by $\phi(n)=g_{t}^{n}(x)$ belongs to $\Phi_{p, q}$.

Let $x, x^{\prime} \in \mathbb{R}$ and $g_{0}(x) \leq x^{\prime} \leq g_{1}(x)$. By the twist property the image of the interval $I=\{x\} \times[0,1]$ intersects the interval $I^{\prime}=\left\{x^{\prime}\right\} \times[0,1]$ at exactly one point, say $\left(x^{\prime}, h\left(x, x^{\prime}\right)\right)$. Let us denote by $T\left(x, x^{\prime}\right)$ the 'triangle' bounded by the bottom boundary component $S_{0}$ of $S$, the interval $I^{\prime}$ and the curve $F(I)$. 


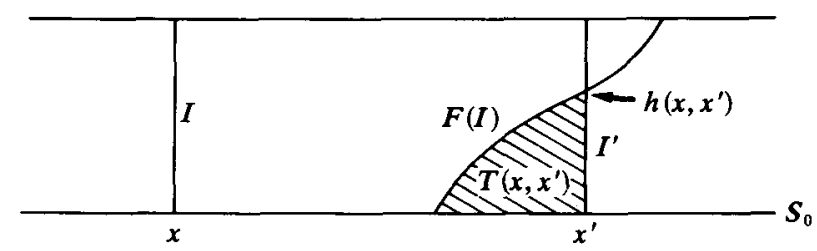

FIGURE 1

Let us define for $\phi \in \Phi_{p, q}$

$$
L_{p, q}(\phi)=\sum_{n=0}^{q-1} \mu(T(\phi(n), \phi(n+p)))
$$

where $\mu$ is the lift to $S$ of the given measure invariant under $f$ and positive on open sets.

We shall need more notation, namely

$$
\begin{aligned}
& h_{1}(n)=h(\phi(n-p), \phi(n)), \\
& \psi_{1}(n)=\left(\phi(n), h_{1}(n)\right), \\
& \psi_{2}(n)=F^{-1} \psi_{1}(n+p) \stackrel{\text { def }}{=}\left(\phi(n), h_{2}(n)\right) .
\end{aligned}
$$

If

$$
h_{1}(n)=h_{2}(n)
$$

then

$$
F \psi_{1}(n)=\psi_{1}(n+p)
$$

so that if (6) is satisfied for every $n$ then the map $\psi_{1}: \mathbb{Z} \rightarrow \mathbb{R}$ defines a periodic orbit with rotation number $p / q$. In fact, it is a Birkhoff orbit of type $(p, q)$. For, otherwise $\phi$ is not strictly monotone and there exists such $n$ that $\phi(n)=\phi(n+1)$ but $h_{1}(n) \neq$ $h_{1}(n+1)$. Since $\phi(n-p) \leq \phi(n-p+1)$ then by the twist property $h_{1}(n)>h_{1}(n+1)$ but then by the twist property again $\phi(n+p)>\phi(n+p+1)$, i.e. $\phi$ is not nondecreasing. Thus, the first statement of the theorem follows from the compactness of $\Phi_{p, q}$ and from the following lemma.

LEMMA. If the functional $L_{p, q}$ reaches its local minimum at $\phi$ then for that $\phi$

$$
h_{1}(n)=h_{2}(n)
$$

for all integer $n$.

Proof. Let us begin with the case when for some $n$

$$
\phi(n-1)<\phi(n)<\phi(n+1) \text { and } h_{1}(n) \neq h_{2}(n) .
$$

Then either

$$
1 \geq h_{1}(n)>h_{2}(n) \geq 0
$$

or

$$
1 \geq h_{2}(n)>h_{1}(n) \geq 0 .
$$


Let us consider the first case. Since

$$
F\left(\phi(n), h_{2}(n)\right)=\left(\phi(n+p), h_{1}(n+p)\right)
$$

it follows from (7) that

$$
h_{1}(n+p)<1 \text {. }
$$

Let us define for $\varepsilon>0$

$$
\tilde{\phi}_{\varepsilon}(m)= \begin{cases}\phi(m) & \text { if } m \neq \neq n(\bmod q), \\ \phi(m)-\varepsilon & \text { if } m=n(\bmod q) .\end{cases}
$$

If $\varepsilon$ is chosen sufficiently small then (7) and (9) guarantee that $\tilde{\phi}_{\varepsilon} \in \Phi_{p, q}$.

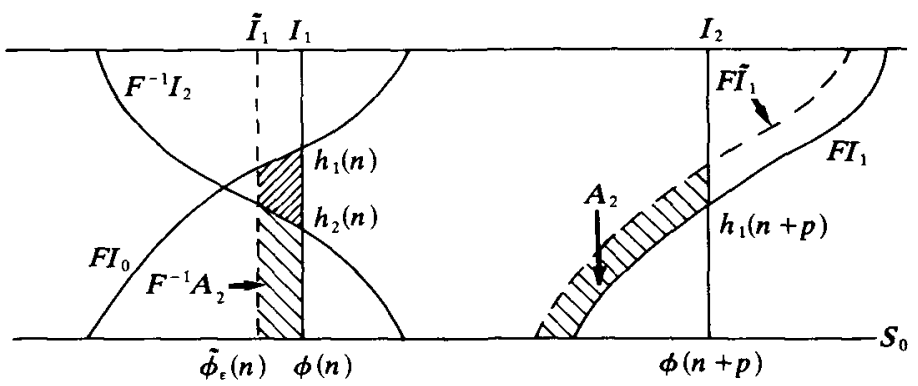

FIGURE 2

Let

$$
\begin{gathered}
I_{0}=\{\phi(n-p)\} \times[0,1], I_{1}=\{\phi(n)\} \times[0,1], \\
I_{2}=\{\phi(n+p)\} \times[0,1], \tilde{I}_{1}=\left\{\tilde{\phi}_{\varepsilon}(n)\right\} \times[0,1] ;
\end{gathered}
$$

$A_{1}$ be the region bounded by $I_{1}, F I_{0}, \tilde{I}_{1}$ and $S_{0}, A_{2}$ be the region bounded by $F I_{1}$, $I_{2}, F \tilde{I}_{1}$ and $S_{0}$.

If $\varepsilon$ is small enough then $F^{-1} A_{2} \subset A_{1}$ so that from $F$-invariance of $\mu$

We have

$$
\mu\left(A_{1}\right)>\mu\left(F^{-1} A_{2}\right)=\mu\left(A_{2}\right) \text {. }
$$

$$
\begin{aligned}
L_{p, q}(\phi)-L_{p, q}\left(\tilde{\phi}_{\varepsilon}\right) & \\
= & \mu(T(\phi(n), \phi(n+p)))+\mu(T(\phi(n-p), \phi(n))) \\
& -\mu(T(\phi(n)-\varepsilon, \phi(n+p)))-\mu(T(\phi(n-p), \phi(n)-\varepsilon)) \\
= & \mu\left(A_{1}\right)-\mu\left(A_{2}\right)>0 .
\end{aligned}
$$

This shows that $\phi$ is not a local minimum. The case when inequality $(8)$ is satisfied is treated similarly but $\tilde{\phi}_{\varepsilon}(n)$ is defined as $\phi(n)+\varepsilon$ (cf. figure 3).

Here $A_{1} \subset F^{-1}\left(A_{2}\right)$.

It remains to consider a more general case when, say

$$
\phi(n-1)<\phi(n)=\phi(n+1)=\cdots=\phi(n+k)<\phi(n+k-1) .
$$

Then by the twist condition

$$
1 \geq h_{1}(n) \geq h_{1}(n+1) \geq \cdots \geq h_{1}(n+k) \geq 0
$$

and

$$
1 \geq h_{2}(n+k) \geq h_{2}(n+k-1) \geq \cdots \geq h_{2}(n) \geq 0 .
$$




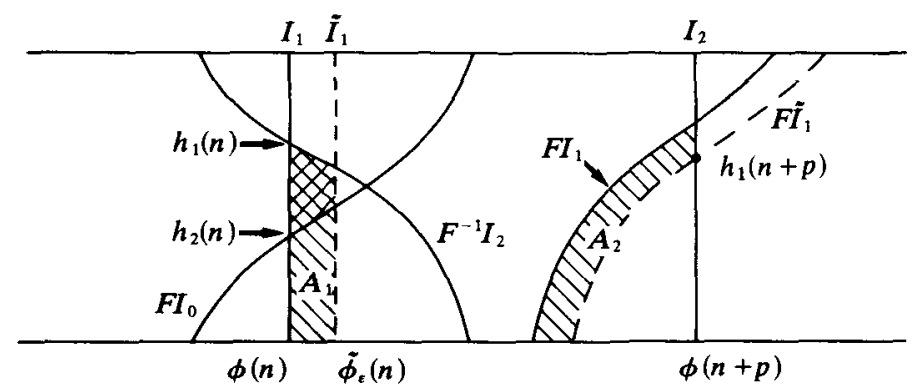

Figure 3

Thus, we have either

$$
1 \geq h_{1}(n)>h_{2}(n) \geq 0
$$

or

$$
1 \geq h_{2}(n+k)>h_{1}(n+k) \geq 0
$$

or

$$
h_{1}(n+l)=h_{2}(n+l), \quad l=0, \ldots, k .
$$

Cases (10) and (11) are considered similarly to (7) and (8) accordingly. It is possible because in the first case $\phi(n)$ is shifted to the left and in the second case $\phi(n+k)$ is shifted to the right so that the monotonicity of $\phi$ is preserved after the perturbation. This completes the proof of the lemma.

Since our proof of Mather's theorem (see §3) does not depend on the second statement of the theorem we shall give only a brief sketch of the proof for that part.

In the smooth case $L_{p, q}$ is differentiable with respect to the smooth structure induced by the embedding into $\mathbb{T}^{q}$. An infinitesimal (and simpler) version of the arguments from the lemma shows that every critical point of $L_{p, q}$ lying inside $\Phi_{p, q}$ must generate a Birkhoff periodic orbit.

Then we take the map $\varphi$ which minimizes the functional $L_{p, q}$ on the space $\Phi_{p, q}$ and consider the space $\Phi_{p, q}^{\phi}$ of all maps $\psi: \mathbb{Z} \rightarrow \mathbb{R}$ satisfying (5) and in addition

$$
\phi(n) \leq \psi(n) \leq \phi(n+1)
$$

for all $n$. The maps $\phi$ and $\tilde{\phi}, \tilde{\phi}(n)=\phi(n+1)$ are critical points (minima) of $L_{p, q}$ on $\Phi_{p, q}^{\phi}$.

By showing that the gradient flow of the function $-L_{p, q}$ maps $\Phi_{p, q}^{\phi}$ into itself we ensure the existence of a critical point $\psi$ which is not a minimum and consequently is different from $\phi$ and $\tilde{\phi}$. This map $\psi$ determines the second Birkhoff periodic orbit.

Smoothness assumptions can be weakened; however, it is absolutely crucial for the argument that the invariant measure is given by a continuous density. I do not see how an argument of that sort can be carried out in full generality.

Birkhoff in [2] obtains the second periodic orbit with rotation number $p / q$ by a sort of index argument but it is not clear why those orbits preserve cyclic order. 


\section{Parabolic and hyperbolic orbits}

Every Mather set $E$ with irrational rotation number carries unique $f$-invariant probability measure $\mu_{E}$. If $f$ is a $C^{1}$ diffeomorphism we can define two Lyapanov characteristic exponents [3] which are constant $\mu_{E^{-}}$almost everywhere. Let $\chi_{1}^{E} \leq \chi_{2}^{E}$ be essential values of those exponents. If $f$ preserves a smooth measure then $\chi_{1}^{E}+\chi \frac{E}{2}=0$ so that there is one non-negative exponent $\chi^{E}$ and one non-positive exponent $-\chi^{E}$.

We shall call a Mather set $E$ hyperbolic if $\chi^{E}>0$ and parabolic if $\chi^{E}=0$.

Problem. Does there exist a $C^{1}\left(C^{2}, C^{\infty}\right.$, real-analytic) area-preserving twist diffeomorphism with a hyperbolic Mather set $E$ ?

Examples of piecewise analytic twist diffeomorphisms for which all Mather's sets are hyperbolic can be obtained from Bunimovich's billiards including his famous stadium-shape example [4]. However, in those examples, apparently Mather's sets intersect the singular set.

Proposition 4. Let $f$ be a $C^{1+\varepsilon}$ twist diffeomorphism preserving a measure $\mu$ equivalent to Lebesgue measure. Then total Lebesgue measure of hyperbolic Mather sets for $f$ is 0 .

Proof. Every point which belongs to a Mather set, but does not belong to a minimal Mather set is not recurrent, i.e. it does not belong to its own $\omega$-limit set. By the Poincaré recurrence theorem the total $\mu$-measure of such points is 0 . Thus, it is enough to consider minimal Mather sets. Those sets are closed and pairwise disjoint. Thus, the partition of $M(f)$ into individual Mather sets is measurable. Every such set has Lebesgue measure 0 as part of a graph of a continuous function. Thus, every ergodic component of $\mu$ on the set $M(f)$ has zero measure. Let us denote the union of hyperbolic Mather sets by $M_{\mathrm{h}}(f) ; f$ has non-zero Lyapanov exponents on that set. By Pesin theory [3] almost every ergodic component on the set with non-zero exponents has positive measure. Thus $\mu\left(M_{\mathrm{h}}(f)\right)=0$.

\section{Concluding remarks}

Mather's theorem about quasi-periodic orbits is a major result in a classical area which up to a certain extent can be put in the same category as celebrated results of Poincaré and Birkhoff on periodic orbits, of Birkhoff and Smale on the orbit structure near homoclinic orbits and of Kolmogorov, Arnold and Moser on the persistence of invariant curves. In particular, Mather's theorem gives an elegant explanation about the 'missing' invariant curves and shows how those curves disappear.

The main purpose of this note is to popularize Mather's result by presenting a more elementary and more geometric proof which works under slightly more general assumptions. More specifically, our method avoids any use of infinitedimensional spaces and involves geometric language (measures, curves, etc.) instead of analytic language used in Mather's original paper (forms, generating functions). Geometric language was first introduced by D. Rudolph when he presented Mather's 
theorem at the 'Twist map' seminar at the University of Maryland in September 1981. In particular, it already followed from Rudolph's presentation that the preservation of area could be replaced by the preservation of some measure positive on open sets.

Another remark: that Mather's set can be obtained as limits of Birkhoff periodic orbits, is mine. It is hard to understand why Birkhoff did not make this remark some $\mathbf{5 0}$ years ago. However, there is at least one vague, but characteristic remark in Birkhoff's paper [5] on p. 254 where he mentions quasi-periodic orbits which must appear as limits of periodic orbits; unfortunately, he does not elaborate.

My secondary goal was to formulate several interesting open problems connected with Mather sets. Beside the problems mentioned in the text I would like to refer to the problem list of the Amherst conference [6] which contains a discussion on the subject.

This paper was written during my visit to Rice University in February 1982. I use this opportunity to thank the Rice Mathematics Department and the Schlumberger foundation for that invitation, and the members of the department for their warm hospitality.

Added in proof. After this paper was written I learnt from M. Herman that a theorem equivalent to Mather's result was proved about two years earlier by $\mathbf{G}$. Aubry. Aubry's results can be found in various preprints. His method is different from both Mather's and mine and looks considerably more complicated than both.

\section{REFERENCES}

[1] J. N. Mather. Existence of quasi-periodic orbits for twist homeomorphisms. Topology. (To appear.)

[2] G. D. Birkhoff. On the periodic motions of dynamical systems. In G. D. Birkhoff Collected Mathematical Papers, vol. 2, pp. 333-353. Amer. Math. Soc., 1950.

[3] Ja. B. Pesin. Characteristic Lyapunov exponents and smooth ergodic theory. Russ. Math. Surveys 32 (1977), 55-112.

[4] L. A. Bunimovich. On the ergodic properties of nowhere dispersing billiards. Comm. Math. Phys. 65 (1979), 295-312.

[5] G. D. Birkhoff. An extension of Poincaré's last geometric theorem. In G. D. Birkhoff Collected Mathematical Papers, vol. 2, pp. 252-266. Amer. Math. Soc., 1950.

[6] A. Katok \& R. Spatzier. Problem list of special session on differential geometry and ergodic theory in Amherst, October 1981. (Preprint.) 